\title{
The effect of intravitreal bevacizumab and ranibizumab on cutaneous tensile strength during wound healing
}

\author{
John B Christoforidis' \\ Jillian Wang ${ }^{2}$ \\ Angela Jiang ${ }^{2}$ \\ James Willard ${ }^{5}$ \\ Cedric Pratt ${ }^{2}$ \\ Mahmoud Abdel-Rasoul ${ }^{3}$ \\ Sashwati Roy ${ }^{4}$ \\ Heather Powell ${ }^{5}$ \\ 'Department of Ophthalmology and \\ Vision Science, College of Medicine, \\ The University of Arizona, Tucson, AZ, \\ USA; ${ }^{2}$ Department of Ophthalmology, \\ College of Medicine, The Ohio State \\ University, Columbus, OH, USA; \\ ${ }^{3}$ Center for Biostatistics, The Ohio \\ State University, Columbus, $\mathrm{OH}$, \\ USA; ${ }^{4}$ Center Surgery, The Ohio \\ State University, Columbus, $\mathrm{OH}$, USA; \\ ${ }^{5}$ Department of Materials Science, \\ College of Engineering, The Ohio \\ State University, Columbus, $\mathrm{OH}$, USA
}

Correspondence: John B Christoforidis Retina Division, Department of Ophthalmology, University of Arizona Medical Center, 655 N Alvernon Way, Suite 108, Tucson, AZ 857II, USA

Tel + I $52032 \mid 3677$

$\mathrm{Fax}+|52032| 3665$

Email jbchristo@hotmail.com
This article was published in the following Dove Press journal:

Clinical Ophthalmology

23 January 2013

Number of times this article has been viewed

Purpose: To investigate the effect of intravitreal bevacizumab and ranibizumab on wound tension and by histopathology during cutaneous wound healing in a rabbit model and to compare this effect to placebo intravitreal saline controls 1 and 2 weeks following intravitreal injection.

Methods: A total of 120 New Zealand white rabbits were randomly assigned to one of three treatment groups each consisting of 40 rabbits. Each group received intravitreal injections of bevacizumab, ranibizumab, or normal saline. Immediately afterwards, each rabbit underwent four $6 \mathrm{~mm}$ full-thickness dermatologic punch biopsies. Twenty rabbits from each agent group underwent wound harvesting on day 7 or day 14. The skin samples were stained for CD34 for vascular endothelial cells on day 7 , and maximal wound tensile load was measured on days 7 and 14. Quantitative assessment of mean neovascularization (MNV) scores was obtained from 10 contiguous biopsy margin $400 \times$ fields of CD34-stained sections by two independent observers.

Results: Wound tension reading means $(\mathrm{N})$ with standard error and adjusted $P$-values on day 7 were: saline placebos, $7.46 \pm 0.87$; bevacizumab, $4.50 \pm 0.88(P=0.041)$; and ranibizumab, $4.67 \pm 0.84(P=0.025)$. On day 14 these were: saline placebos, $7.34 \pm 0.55$; bevacizumab, $6.05 \pm 0.54(P=0.18)$; and ranibizumab $7.99 \pm 0.54(P=0.40)$. MNV scores in CD34 stained sections were: saline controls, $18.31 \pm 0.43$; bevacizumab, $11.02 \pm 0.45(P<0.0001)$; and ranibizumab, $13.55 \pm 0.43(P<0.0001)$. The interobserver correlation coefficient was 0.928 .

Conclusion: At day 7, both anti-vascular endothelial growth factor (anti-VEGF) agents had significantly suppressed MNV scores and exerted a significant reduction of cutaneous wound tensile strength compared with saline controls. At day 14, neither agent produced a significant effect on tensile wound strength. Since angiogenesis is an integral component of the proliferative phase of wound healing, we encourage clinicians to be aware of their patients' recent surgical history during intravitreal anti-VEGF therapy and to consider refraining from their use during the perioperative period.

Keywords: wound healing, tensile strength, bevacizumab, ranibizumab

\section{Introduction}

Bevacizumab and ranibizumab are two therapeutic agents that suppress all isoforms of vascular endothelial growth factor (VEGF). They are currently the most commonly used agents for the treatment of the exudative form of age-related macular degeneration (AMD) and macular edema due to central and branch retinal vein occlusion. Both agents are used on an off-label basis for the treatment of non-AMD choroidal neovascularization and diabetic macular edema in the United States.

Wound healing complications following systemic use of bevacizumab are well known and include wound dehiscence and hemothorax after thoracotomy and port 
placement, surgical site bleeding, incisional hernias, erosions, ecchymoses, and infection. ${ }^{1-5}$ These adverse events are especially prevalent in patients over the age of 60 years. ${ }^{6}$ As a result of these, several reports have recommended discontinuation of systemic bevacizumab 4-6 weeks prior to surgery and recommencement 4 weeks after surgery. ${ }^{6-10}$ Despite these findings, there has been little investigation into the effects of wound healing after intravitreal use of bevacizumab and ranibizumab. This is partly because of the large number of variables associated with wound healing (eg, differing incision size and depth, time of wounding in relation to intravitreal injection). Therefore, an animal model in which the number of these variables is minimized becomes a reasonable option to study the effects of intravitreal antiVEGF agents on cutaneous wound healing.

A recent histopathologic report demonstrated that both of these intravitreally placed agents suppressed neovascularization of cutaneous wound margins by quantitative assessment of CD34 immunostaining 1 week following treatment compared with untreated controls. There was a nonsignificant inhibitory trend for both bevacizumab and ranibizumab $(P=0.013$ and 0.071 respectively, alpha $<0.008)$. At 2 weeks, there was no demonstrable effect for either agent ( $P=0.471$ and 0.297 respectively), presumably since the wounds had entered the maturation phase of healing when the number of wound blood vessels is decreased. ${ }^{11}$

To the authors' knowledge, there are no reports on the functional outcomes of cutaneous wound healing following intravitreal anti-VEGF therapy. Measurement of wound tensile strength is an established method for evaluating the biomechanical properties of wound repair, specifically collagen function. ${ }^{14-17}$ The primary purpose of this study was to investigate the effect of intravitreally placed bevacizumab and ranibizumab on wound tensile strength during cutaneous wound healing in a rabbit model at 1 and 2 weeks and to compare these effects to intravitreal saline controls. Since there was an inhibitory trend at week one in our previous report, we also evaluated neovascularization of cutaneous wound margins by quantitative assessment of CD34 immunostaining 1 week following treatment compared with untreated controls.

\section{Methods}

\section{Intravitreal injection and cutaneous biopsy}

A total of 120 New Zealand white rabbits (Myrtle's Rabbitry, Thompsons Station, TN, USA) weighing 3.8-3.9 kg was used in this study. All treatments were conducted in agreement with the principles of laboratory animal care (National Institutes of Health publication No 85-23, revised 1985), the OPRR Public Health Service Policy on the Humane Care and Use of Laboratory Animals (revised 1986) and the US Animal Welfare Act. All experimental protocols were approved, and the procedures followed were in accordance with the ethical standards of The Institutional Animal Care and Use Committee at The Ohio State University. Prior to general anesthesia, each rabbit was administered $0.3 \mathrm{mg} / \mathrm{kg}$ of meloxicam orally. Anesthesia was induced with $10 \mathrm{mg} / \mathrm{mL}$ acepromazine subcutaneously and maintained with 3\%-5\% isoflurane. Electric clippers were used to shave the dorsal skin of the rabbit, and this area was then cleansed with $10 \%$ povidone-iodine and 70\% alcohol. A standard $6 \mathrm{~mm}$ dermatologic punch biopsy (Sklar Tru Punch; Sklar Instruments, West Chester, PA, USA) was used to create two full-thickness cutaneous wounds with removal of the skin core. These were created approximately $3 \mathrm{~cm}$ lateral to each side of the cervicothoracic vertebral column in the mid-dorsum area. The rabbits were randomly assigned to one of three groups, each consisting of 40 rabbits. Immediately following the biopsies, each rabbit received an intravitreal injection in the left eye consisting of $0.05 \mathrm{~mL}$ of $1.25 \mathrm{mg} / 0.05 \mathrm{~mL}$ bevacizumab, $0.5 \mathrm{mg} / 0.05 \mathrm{~mL}$ ranibizumab or $0.05 \mathrm{~mL}$ of normal saline.

\section{Wound biomechanics}

To assess the strength of the healing wounds, skin biopsies $(30 \mathrm{~mm} \times 80 \mathrm{~mm})$ were harvested from the flanks of the bevacizumab-, ranibizumab-, and saline-treated rabbits 7 or 14 days post wounding (Figure 1A). Only biopsy sites with clearly visible central core defects were harvested. Following wound harvesting, the anesthetized rabbits were euthanized by intravenous injection of $3 \mathrm{~mL}$ of saturated potassium chloride. Each rectangular-shaped skin biopsy was stored in a $50 \mathrm{~mL}$ polypropylene conical tube containing normal saline solution and placed in ice for 2-4 hours until cut into a dog bone-shaped specimen it was (gauge length of $2 \mathrm{~mm}$ and gauge width of $4 \mathrm{~mm}$ ) for tensile testing (Figure 1B). The harvested skin was placed in a position such that the biopsied site was in the center of the gauge length and width (Figure 1B). The muscle fascia was removed from each dog bone specimen with a scalpel. Each specimen was then mounted into the grips of a TestResources mechanical tester model 1000R12 (TestResources, Shakopee, MN, USA) with a $50 \mathrm{lbf}$ load cell (Figure 1C). All skin samples were strained at a rate of $2 \mathrm{~mm} / \mathrm{s}$ until failure and maximum load was recorded in newtons $(\mathrm{N})$. Specimens that failed at the wound site due to the weight of the bottom half of the dog bone were assigned a strength of zero $\mathrm{N}$. 


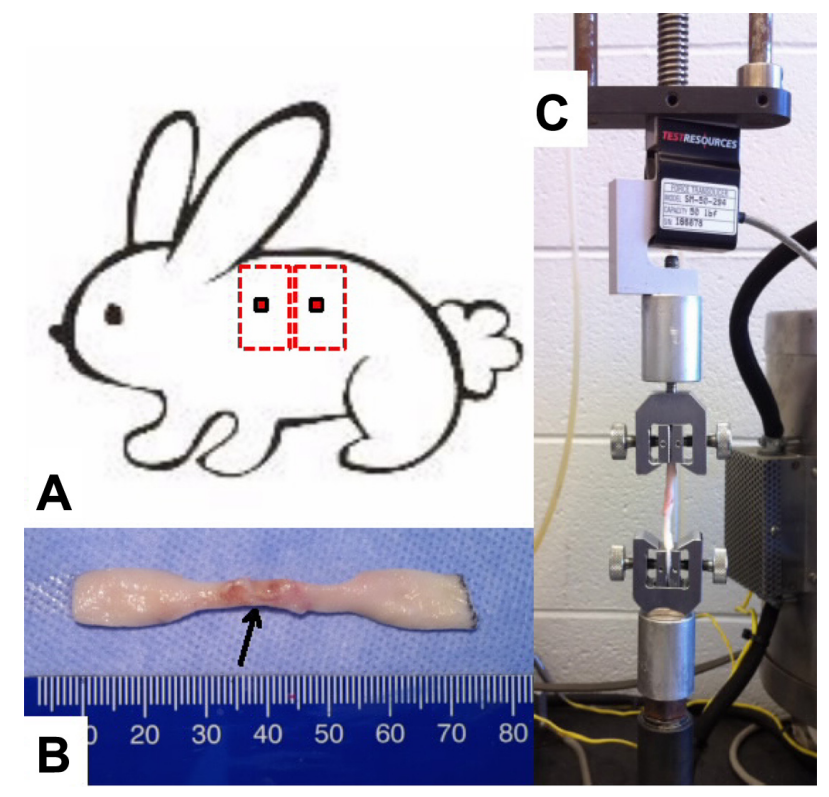

Figure I To assess the strength of the healing wounds, skin biopsies $(30 \mathrm{~mm} \times 80 \mathrm{~mm})$ were harvested from the flanks of the bevacizumab-, ranibizumab-, and saline-treated rabbits 7 or 14 days post wounding (A). Following wound harvesting, the anesthetized rabbits were euthanized by intravenous injection of $3 \mathrm{~mL}$ of saturated potassium chloride. Each rectangular-shaped skin biopsy was stored in a $50 \mathrm{~mL}$ polypropylene conical tube containing normal saline solution and placed in ice for 2-4 hours until they were cut into dog bone-shaped specimens (gauge length of $2 \mathrm{~mm}$ and gauge width of $4 \mathrm{~mm}$ ) for tensile testing (B). The harvested skin was placed in a position such that the biopsied site was in the center of the gauge length and width. The muscle fascia was removed from each dog bone specimen with a scalpel. Each specimen was then mounted into the grips of a TestResources mechanical tester model I000RI2 (TestResources, Shakopee, MN, USA) with a 50 lbf load cell (C).

\section{Histopathology}

On day 7, two $6 \mathrm{~mm}$ biopsy sites were harvested from the dorsal skin of the anesthetized rabbit, with a standard $12 \mathrm{~mm}$ dermatologic punch biopsy (Accuderm, Ft Lauderdale, FL, USA) that included the entire previous biopsy site along with surrounding normal skin. Only biopsy sites with clearly visible central core defects were harvested. Immediately after harvesting, skin samples were placed into conical tubes containing 10\% neutral-buffered formalin. After 48 hours, the formalin was exchanged with $70 \%$ ethanol and the biopsied skin was routinely processed, embedded in paraffin wax, and sectioned at $5 \mu \mathrm{m}$. Serial paraffin sections placed on poly-Llysine slides were immunohistochemically stained for CD34 as described in a previous report. ${ }^{11}$ Ten contiguous $400 \times$ fields at the biopsy site wound margins of CD34-stained sections were photographed using a six-headed Olympus BX51 light microscope with attached Altra 20 digital camera and MicroSuite software (B\&B Microscopes Limited, Pittsburgh, PA, USA). The photomicrographs were assorted randomly, and two independent observers who were blinded to the experimental groups recorded the numbers of CD34-stained endothelial cell clusters for each slide. The observer score means for each photomicrograph and the mean neovascularization (MNV) scores representing all of the 10 photomicrographs for each subject were calculated and evaluated.

\section{Statistical analysis}

A linear mixed model with random intercepts was fit to compare the difference in max load between treatment groups and the control group. The mixed model adjusts for the possible correlation of repeated measurements within subjects. Holm's step-down method for multiple comparisons was used to adjust for multiple hypothesis tests.

A linear mixed model was fit to compare the difference in mean scores between all three treatment groups (avastin, lucentis, and saline). The mixed model adjusts for the possible correlation of repeated measurements of specimens within wounds and wounds within rabbits by treating wounds within rabbits and specimens within wounds as random effects. Tukey's method for pair-wise comparisons was used to adjust for multiple hypothesis tests. Interobserver correlation coefficient was used to assess agreement among raters.

\section{Results Wound biomechanics}

Following the procedures, the rabbits were monitored daily. At the time of wound harvesting all of the rabbits had clearly evident cutaneous wound without signs of infection. The treated eyes were absolutely quiet without signs of discharge, redness, or irritation at the time of harvesting.

All of the subjects had at least one specimen harvested for tensile strength evaluation. However, eight biopsy sites on day 7 (five bevacizumab and three saline controls) from eight separate rabbits and one biopsy site from on day 14 (saline control) did not have clearly visible central core defects and these were not harvested.

On day 7 , both treatment groups had significantly reduced wound tensile strength compared with saline controls. The following wound tension reading means with standard error and $95 \%$ confidence intervals (CIs) and adjusted $P$-values in newtons were found: saline placebos, $7.46 \pm 0.87$, CI 5.72-9.20; bevacizumab, $4.50 \pm 0.88$, CI 2.74-6.27 $(P=0.0406)$; and ranibizumab, $4.67 \pm 0.84$, CI 2.97-6.37 $(P=0.0250)$. On day 14 , the findings were not significant for either group. Compared with saline controls $(7.34 \pm 0.55$, CI 6.24-8.44), bevacizumab (6.05 \pm 0.54 , CI 4.97-7.14) $(P=0.181)$ remained reduced, while ranibizumab tensile strength $(7.99 \pm 0.54$, CI $6.91-9.08)(P=0.402)$ was elevated compared with both groups. Figure 2 presents a graphic summary of the findings for day 7 and day 14 . 


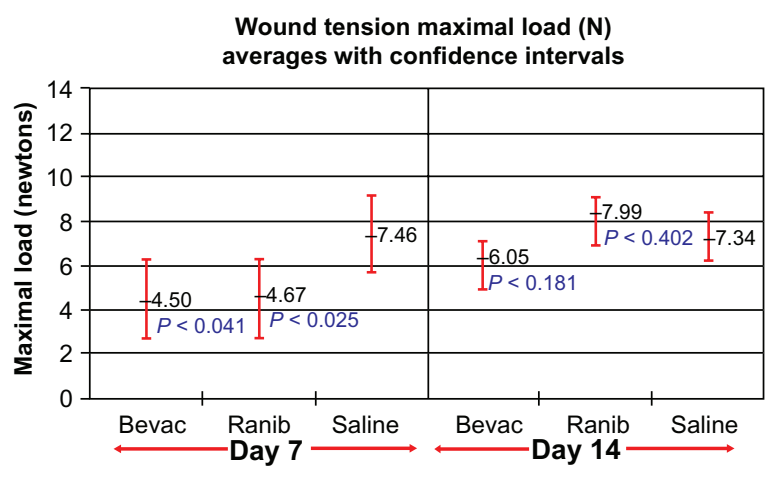

Figure 2 Wound tension maximal load means with $95 \%$ confidence intervals and treatment group $P$-values for each group at days 7 and 14 .

Note: The adjusted $P$-values are in relation to saline control at each time point.

Abbreviations: Bevac, bevacizumab-treated group; Ranib, ranibizumab-treated group; Saline, saline-treated group.

\section{Histopathology}

At the time of wound harvesting on day 7,115/120 wound sites were clearly visible with central core defects and were harvested. All of the subjects had at least one biopsied wound harvested for histopathologic evaluation. Five biopsy sites (three bevacizumab, one ranibizumab, and one saline control) from five separate rabbits did not have clearly visible central core defects and were not harvested. On light microscopy, all of the wound margin borders were clearly visible and were scored. MNV scores were calculated from all of the $400 \times$ CD34-stained rabbit skin sections (Figure 3).

Both of the treatment groups were found to significantly inhibit the number of vessels compared with the placebo group (shown as mean with standard deviation and 95\% CI). Compared with saline controls (18.31 \pm 0.43 , CI 17.45-19.16), bevacizumab $(11.02 \pm 0.45$, CI 10.13-11.91) $(P<0.0001)$ inhibited neovascularization in cutaneous wound margins. The mean MNV for ranibizumab was higher than bevacizumab and was significantly higher than saline control (13.55 \pm 0.43 , CI 12.70-14.41; $P<0.0001$ ) (Figure 4). The interobserver correlation coefficient was 0.921 , indicating high interpretation agreement between raters $(P<0.0001)$. Score 1 and score 2 were highly correlated, with a correlation coefficient of 0.928 . The mean of score 1 and score 2 was used as the outcome variable to compare the treatment effects.

\section{Discussion}

In this study, both intravitreal bevacizumab and ranibizumab were found to exert significant inhibition on cutaneous wound tensile strength and neovascularization in cutaneous wound margins at day 7 compared with saline controls. At day 14 , neither agent produced a significant effect on tensile wound strength. This work demonstrates that intravitreally placed bevacizumab and ranibizumab suppresses cutaneous wound tensile strength during the proliferative phase of wound healing in a rabbit model.

The proliferation phase during wound healing usually occurs between days 4 and $14 .{ }^{18}$ Angiogenesis suppression at this phase disturbs the integrity of wound restoration. ${ }^{19-21}$ This effect on cutaneous wound healing is the likely mechanism by which systemic bevacizumab adversely affects wound healing as previously described in several clinical studies. ${ }^{6,8-10,22-24}$ Following the proliferative phase of wound healing is the maturation phase, when the number of blood vessels in the wound decreases.

The suppressed histologic wound changes on day 7 translated to functional cutaneous changes as demonstrated

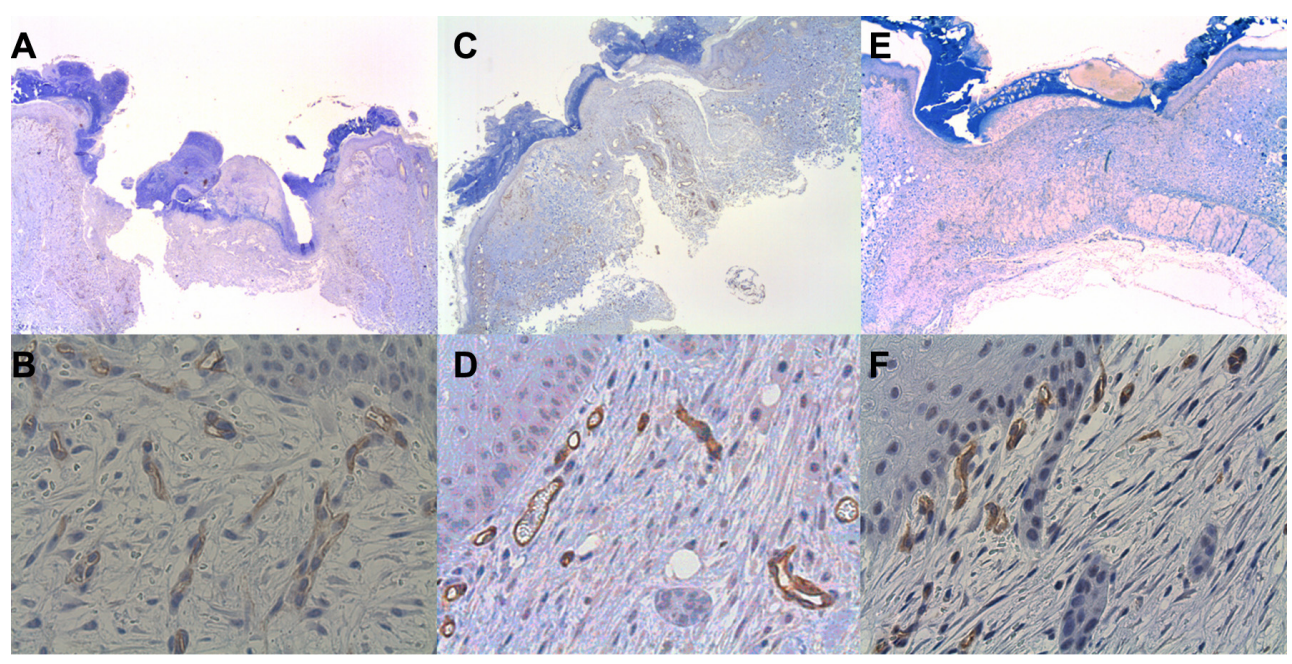

Figure 3 Representative CD34 histological figures of cutaneous wounds I week after wounding. At 20×, wound margins are demonstrated (A, C and E). At 400×, note increased numbers of endothelial cells counts in the placebo (about I7) (B) versus bevacizumab (about II) (D) and ranibizumab (about I2) (F). 
Day 7 - NV means with $95 \%$ confidence intervals

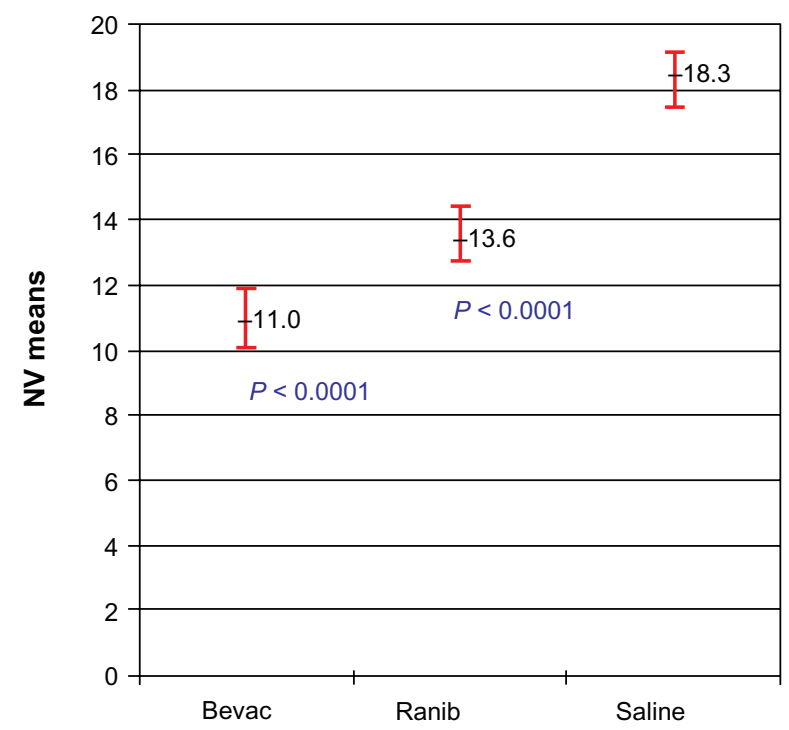

Figure 4 CD34 endothelial cell cluster per high power field means with $95 \%$ confidence intervals and treatment group $P$-values for each group at day 7 . Abbreviations: NV, neovascularization; Bevac, bevacizumab-treated group; Ranib, ranibizumab-treated group; Saline, saline-treated group.

by wound tension measurements. On day 14 , differences in wound tensile strength between groups were not significant, and the number of vessels was decreased compared with those on day 7. The findings in this study are consistent with a recent report describing an inhibitory trend for MNV scores on day 7 for bevacizumab $(P=0.013)$ and ranibizumab $(P=0.071)$ compared with pegaptanib $(P=0.378)$ and untreated controls. ${ }^{11}$ In that report, the central cores were not removed and the average MNV scores were lower for all groups than in the present study.

In addition to the effects on wound healing, low rates of hypertension and arterial thromboembolic events including angina pectoris, myocardial infarction, transient ischemic attack, and cerebrovascular accident have been associated with both bevacizumab and ranibizumab. ${ }^{25-28}$ Because of the small incidences of these events reported in these studies, they do not have enough statistical power to detect low rates of these events. ${ }^{29}$ At this time, the mechanism of serum availability of bevacizumab and ranibizumab after intravitreal injection is speculative. It may gain access to the systemic circulation either anteriorly through the trabecular meshwork or posteriorly through the retina and the choroidal circulation. The effects of bevacizumab and ranibizumab on wound healing following intravitreal injections are yet to be specifically examined in a major clinical trial involving these agents.

There were several limitations in this study. The use of a rabbit model has several inherent constraints. The vitreous volume in rabbits is approximately one-third that of humans ( $4.5 \mathrm{~mL}$ versus $1.5 \mathrm{~mL}$ ), while the serum compartment is significantly smaller than that of humans. Clinical doses of intravitreal anti-VEGF agents in a rabbit model increase the systemic exposure of these agents in comparison with humans. The clearance half-lives of bevacizumab and ranibizumab have been found to be longer in adult humans than in rabbits, possibly because the larger vitreous volume results in lowered agent concentrations and increased diffusion time prior to clearance from the eye. ${ }^{30-33}$ On the other hand, the rabbit vitreous volume is closer in size to that of a retinopathy of a prematurity eye $(1.6-2.5 \mathrm{~mL})^{34,35}$ and may be used to more accurately simulate the intravitreal pharmacokinetic properties of these agents in this group of patients. Furthermore, rabbit retinas contain a tapetum lucidum, and it is unclear how this affects agent absorption. Nevertheless, the rabbit model is an accepted and valuable experimental model for many human ocular and systemic conditions that otherwise could not be feasibly studied.

Each rabbit had at least one biopsied wound harvested for tensile and histopathologic evaluation. Eight wounds from day 7 , and 1 wound from day 14 had incomplete central core defects and were excluded from the tensiometry data measurements. With incomplete core removal from the wound, normal skin tissue overgrowth occurs and dog bone wound tensiometry is measured high. This is a known limitation with tensile strength measurements in immature wounds and was an important reason that two wounds were created per subject. Similarly, five wound biopsies for histopathology were not harvested because of incomplete central core defects. Incomplete core defects result in a decreased inflammatory response and lowered endothelial cell proliferation. In a previous report where central cores were not removed, MNV scores for bevacizumab were 7.41 (versus 10.91), ranibizumab 8.71 (versus 13.51), and 11.51 (versus 18.37) for the controls. Lastly, we were, unfortunately, not able to directly measure ranibizumab and bevacizumab in the blood because the methodology was not available at our institution or any of our referral labs.

Rabbit VEGF has been found to bind to bevacizumab and is estimated to be $94 \%$ homologous with human counterparts of VEGF 121, 165, and 189 isoforms. ${ }^{36,37}$ Nevertheless, because of interspecies differences, it is somewhat presumptuous to extrapolate our findings in this rabbit model to clinical situations with humans. Clinicians should be aware of the potential for delayed wound healing, to be cognizant of their patients' recent surgical history and/or the presence of any open wounds, particularly in retinopathy of prematurity 
patients. Observation or the use of photodynamic therapy could be used as an alternative form of treatment during this period of time.

In summary, in the present study, intravitreal bevacizumab and ranibizumab were found to significantly suppress the wound tensile strength and neovascularization in cutaneous wound margins 1 week following treatment when compared with saline-treated placebo controls. Because of the increasing number of patients undergoing intravitreal anti-VEGF therapy, further studies of the effects of intravitreally placed anti-VEGF agents on the skin and other organ systems is warranted.

\section{Acknowledgment}

The National Center for Research Resources, National Institutes of Health, Award Number UL1RR025755. The content is solely the responsibility of the authors and does not necessarily represent the official views of the National Center for Research Resources or the National Institutes of Health.

\section{Disclosure}

None of the authors have any conflicts of interest that pertain to the information presented in this manuscript.

\section{References}

1. Erinieri JP, Fong AJ, Kemeny NE, Brown KT, Getraidman GI, Solomon SB. Timing of administration of bevacizumab chemotherapy affects wound healing after chest wall port placement. Cancer. 2011;117(6): 1296-1301.

2. Rosamond W, Flegal K, Furie K, et al. Heart disease and stroke statistic 2008 Update: a report from the American Heart Association Statistics Committee and Stroke Statistics Subcommittee. Circulation. 2008;117(4); e25-e146.

3. Rogers S, McIntosh RL, Cheung N, et al; for the International Eye Disease Consortium. The prevalence of retinal vein occlusion: pooled data from population studies from the United States, Europe, Asia, and Australia. Ophthalmology. 2010;117(2):313-319. e1.

4. Yang JC, Haworth L, Sherry RM, et al. A randomized trial of bevacizumab, an anti-vascular endothelial growth factor antibody, for metastatic renal cancer. N Engl J Med. 2003;359(5):427-434.

5. Pouessel D, Culine S. High frequency of intracerebral hemorrhage in metastatic renal carcinoma patients with brain metastases treated with tyrosine kinase inhibitors targeting the vascular endothelial growth factor receptor. Eur Urol. 2008;53(2):376-381.

6. Allegra CJ, Yothers G, O’Connell MJ, et al. Initial safety report of NSABP C-08: a randomized Phase III study of modified FOLFOX6 with or without bevacizumab for the adjuvant treatment of patients with stage II or III colon cancer. J Clinic Oncol. 2009;27(20):3385-3390.

7. Gordon CR, Rojavin Y, Patel M, et al. A review on bevacizumab and surgical wound healing: an important warning to all surgeons. Ann Plast Surg. 2009;62(6):707-709.

8. Zawacki WJ, Walker TG, DeVasher E, et al. Wound dehiscence or failure to heal following venous access port placement in patients receiving bevacizumab therapy. J Vasc Interv Radiol. 2009;20(5):624-627.

9. Thornton AD, Ravn P, Winslet M, Chester K. Angiogenesis inhibition with bevacizumab and the surgical management of colorectal cancer. $\mathrm{Br}$ J Surg. 2006;93(12):1456-1463.
10. Scappaticci FA, Fehrenbacher L, Cartwright T, et al. Surgical wound healing complications in metastatic colorectal cancer patients treated with bevacizumab. J Surg Oncol. 2005;91(3):173-180.

11. Christoforidis JB, Rickets R, Pratt C, et al. The effect of intravitreal anti-VEGF agents on peripheral wound healing in a rabbit model. Clin Ophthalmol. 2012;6:61-69.

12. Reid RR, Said HK, Mogford JE, Mustoe TA. The future of wound healing: pursuing surgical models in transgenic and knockout mice. J Am College of Surgeons. 2004;199(4):578-585.

13. Gottrup F, Agren MS, Karlsmark T. Models for use in wound healing research: a survey focusing on in vitro and in vivo adult soft tissue. Wound Repair Regen. 2000;8(2):83-96.

14. Beck LS, Chen TL, Mikalauski P, Ammann AJ. Recombinant human transforming growth factor-beta 1 (rhTGF-beta 1) enhances healing and strength of granulation skin wounds. Growth Factors. 1990;3(4): 267-275.

15. Gould LJ, Leong M, Sonstein J, Wilson S. Optimization and validation of an ischemic wound model. Wound Repair Regen. 2005;13(6):576-582.

16. Gupta A, Jain GK, Raghubir. A time course study for the development of an immunocompromised wound model, using hydrocortisone. J Pharm Toxicol Methods. 1999;41(4):183-187.

17. Matsuda H, Koyama H, Sato H, et al. Role of nerve growth factor in cutaneous wound healing: accelerating effects in normal and healingimpaired diabetic mice. J Exp Med. 1998;187(3):297-306.

18. Howdieshell TR, Riegner C, Gupta V, Callaway D, Sathyanarayana McNeil PL. Normoxic wound fluid contains high levels of vascular endothelial growth factor. Ann Surg. 1998;228:707-715.

19. Hunt T. Wound Healing and Wound Infection: theory and surgical practice. New York, NY: Appleton-Century-Crofts; 1980.

20. Brown LF, Van De Water L, Harvery VS, Dvorak HF. Fibrinogen influx and accumulation of cross-linked fibrin in healing wounds and in tumor stroma. Am J Pathol. 1988;130:455-465.

21. Breuing K, Eriksson E, Liv P, Miller DR. Healing of partial thickness porcine skin wounds in a liquid environment. J Surg Res. 1992;52:50-58.

22. Bose D, Meric-Bernstam F, Hofstetter W, Reardon DA, Flaherty KT, Ellis LM. Vascular endothelial growth factor targeted therapy in the perioperative setting: implications for patient care. Lancet Oncol. 2010;11(4):373-382.

23. Ellis LM, Curley SA, Grothey A. Surgical resection after downsizing of colorectal liver metastasis in the era of bevacizumab. J Clinic Oncol. 2005;23:4853-4855.

24. Sugrue MM, Purdie DM, Feng S, et al. Serious wound healing complications (sWHC) following surgery in patients (pts) with metastatic colorectal cancer (mCRC) receiving bevacizumab (BV): results from the BRiTE observational cohort study (OCS). J Clin Oncol. 2008;26(Suppl 15):abstr 4105.

25. Howdieshell TR, Callaway D, Webb WL, et al. Antibody neutralization of vascular endothelial growth factor inhibits wound granulation tissue formation. J Surg Res. 2001;96:173-182.

26. Rich RM, Rosenfeld PJ, Pulafito CA, et al. Short-term safety and efficacy of intravitreal bevacizumab (Avastin) for neovascular age-related macular degeneration. Retina. 2006;26:495-511.

27. Michels S, Rosenfeld PJ, Puliafito CA, Marcus EN, Venkatraman AS. Systemic bevacizumab (Avastin) therapy for neovascular age-related macular degeneration twelve-week results of an uncontrolled open-label clinical study. Ophthalmology. 2005;112(6):1035-1047.

28. Martin DF, Maguire MG, Ying GS, et al; for the CATT Research Group. Ranibizumab and bevacizumab for neovascular age-related macular degeneration. N Engl J Med. 2011;364(20):1897-1908.

29. Csaky K, Do DV. Safety implications of vascular endothelial growth factor blockade for subjects receiving intravitreal anti-vascular endothelial growth factor therapies. Am J Ophthalmol. 2009;148(5):647-656.

30. Gaudreault J, Webb W, Van Hoy M, et al. Pharmacokinetics and retinal distribution of AMD rhufab V2 after intravitreal administration in rabbits. AAPS Pharm Sci. 1999;Suppl 1:3207. 
31. Zhu Q, Ziemssen F, Henke-Fahle S, et al. Vitreous levels of bevacizumab and vascular endothelial growth factor- $\alpha$ in patients with choroidal neovascularization. Ophthalmology. 2008;115:1750-1755.

32. Krohne TU, Eter N, Holz FG, Meyer CH. Intraocular pharmacokinetics of bevacizumab after a single intravitreal injection in humans. Am J Ophthlamol. 2008;146:508-512.

33. Maurice DM, Mishima S. Pharmacology of the Eye. New York: SpringerVerlag; 1984.

34. Darlow BA, Ells AL, Gilbert CE, Gole GA, Quinn GE. Are we there yet? Bevacizumab therapy for retinopathy of prematurity. Arch Dis Child Fetal Neonatal Ed. Epub December 30, 2011.
35. Meyer CH, Krohne TU, Holz FG. Intraocular pharmacokinetics after a single intravitreal injection of $1.5 \mathrm{mg}$ versus $3.0 \mathrm{mg}$ of bevacizumab in humans. Retina. 2011;31:1877-1884.

36. Lin YS, Nguyen C, Medoza JL, et al. Preclinical pharmacokinetics, interspecies scaling and tissue distribution of a humanized monoclonal antibody against vascular endothelial growth factor. J Pharmacol Exp Ther. 1999;299:371-378.

37. Watkins RH, D'Angio CT, Ryan RM, Patel A, Maniscalco WM. Differential expression of VEGF mRNA splice variants in newborn and adult hyperoxic lung injury. Am J Physiol Lung Cell Mol Physiol. 1999;276:858-867.
Clinical Ophthalmology

\section{Publish your work in this journal}

Clinical Ophthalmology is an international, peer-reviewed journal covering all subspecialties within ophthalmology. Key topics include: Optometry; Visual science; Pharmacology and drug therapy in eye diseases; Basic Sciences; Primary and Secondary eye care; Patien Safety and Quality of Care Improvements. This journal is indexed on

Submit your manuscript here: http://www.dovepress.com/clinical-ophthalmology-journal

\section{Dovepress}

PubMed Central and CAS, and is the official journal of The Society of Clinical Ophthalmology (SCO). The manuscript management system is completely online and includes a very quick and fair peer-review system, which is all easy to use. Visit http://www.dovepress.com/ testimonials.php to read real quotes from published authors. 